\title{
Multidimensional Rank Reduction Estimator for Parametric MIMO Channel Models
}

\author{
Marius Pesavento \\ Lehrstuhl für Signaltheorie, Ruhr-Universität Bochum, 44780 Bochum, Germany \\ Email:mps@sth.rub.de \\ Christoph F. Mecklenbräuker \\ FTW - Forschungszentrum Telekommunikation Wien, 1220 Wien, Austria \\ Email: cfm@ftw.at

\section{Johann F. Böhme} \\ Lehrstuhl für Signaltheorie, Ruhr-Universität Bochum, 44780 Bochum, Germany \\ Email: boehme@sth.rub.de
}

Received 28 May 2003; Revised 25 November 2003

\begin{abstract}
A novel algebraic method for the simultaneous estimation of MIMO channel parameters from channel sounder measurements is developed. We consider a parametric multipath propagation model with $P$ discrete paths where each path is characterized by its complex path gain, its directions of arrival and departure, time delay, and Doppler shift. This problem is treated as a special case of the multidimensional harmonic retrieval problem. While the well-known ESPRIT-type algorithms exploit shiftinvariance between specific partitions of the signal matrix, the rank reduction estimator (RARE) algorithm exploits their internal Vandermonde structure. A multidimensional extension of the RARE algorithm is developed, analyzed, and applied to measurement data recorded with the RUSK vector channel sounder in the $2 \mathrm{GHz}$ band.
\end{abstract}

Keywords and phrases: array processing, rank reduction, MIMO, channel sounder, ESPRIT.

\section{INTRODUCTION}

Multidimensional harmonic retrieval problems arise in a large variety of important applications like synthetic aperture radar, image motion estimation, chemistry, and doubledirectional channel estimation for multiple-input multipleoutput (MIMO) communication systems [1]. Also certain signal separation problems can be solved under this framework.

The 4D parameter estimation problem for MIMO channel sounder measurements applies to the following doubledirectional MIMO channel model in which the signal is assumed to propagate from the transmitter to the receiver over $P$ discrete propagation paths. Each path $(p=1, \ldots, P)$ is characterized by the following parameters: complex path gain $w_{p}$, direction of departure (DOD) $\theta_{p}$, direction of arrival (DOA) $\phi_{p}$, propagation delay $\tau_{p}$, and Doppler shift $\nu_{p}$.

We assume an idealized data acquisition model for MIMO channel sounders. In this model, data consists of simultaneous measurements of the individual complex baseband channel impulse responses between all $M$ transmit antenna elements and all $L$ receive antenna elements after ideal lowpass filtering. These are assembled in a 3-way array with dimensions $K \times L \times M$. Such a 3-way array is in the following referred to as a "MIMO snapshot" and consists of $K$ time samples at sampling period $T_{s}$. A MIMO snapshot is acquired in a duration $T_{a}$. We repeat $N$ MIMO snapshot measurements consecutively in time and assemble a 4-way array of dimensions $K \times L \times M \times N$ which we refer to as a "Doppler block." We assume that all path parameters $w_{p}$, $\theta_{p}, \phi_{p}, \tau_{p}, v_{p}$ remain constant within the acquisition time $N T_{a}$ of each Doppler block. Individual Doppler blocks are indexed by $i=1, \ldots, J$. Between any two Doppler blocks, the complex path gain $w_{p}$ may vary arbitrarily while the remaining path parameters are constant for $p=1, \ldots, P$. In Section 5.2, we describe the data acquisition with MEDAV's RUSK-ATM channel sounder [2] (http://www.medav.de), (http://www.channelsounder.de) which was used for the experiment.

The $i$ th Doppler block is modelled as

$$
\begin{aligned}
x_{k, \ell, m, n}(i)= & \sum_{p=1}^{P} w_{p}(i) \operatorname{sinc}\left(k-\frac{\tau_{p}}{T_{s}}\right) b_{p}^{\ell} c_{p}^{m} d_{p}^{n} \\
& + \text { noise }
\end{aligned}
$$


where

$$
\begin{gathered}
b_{p}=e^{-j\left(2 \pi d_{R} / \lambda\right) \cos \phi_{p}}, \quad c_{p}=e^{-j\left(2 \pi d_{T} / \lambda\right) \cos \theta_{p}}, \\
d_{p}=e^{-j(2 \pi / N) v_{p}} .
\end{gathered}
$$

The first index $k$ represents the time sample, the second index $\ell$ represents the Rx element number, the third index $m$ represents the Tx element number, and the fourth index $n$ represents the Doppler block number. We have assumed uniform linear receive and transmit arrays, $\lambda$ is the wavelength, $d_{R}$ and $d_{T}$ denote the elemental spacings of the receive and transmit side, respectively.

After a discrete Fourier transform over the time sample index $k$, we obtain

$$
\begin{gathered}
y_{k, \ell, m, n}(i)=\sum_{p=1}^{P} w_{p}(i) a_{p}{ }^{k} b_{p}{ }^{\ell} c_{p}{ }^{m} d_{p}{ }^{n}+\text { noise } \\
i=1, \ldots, J, \quad k=1, \ldots, K \\
\ell=1, \ldots, L, \quad m=1, \ldots, M \\
n=1, \ldots, N
\end{gathered}
$$

where

$$
\begin{aligned}
& a_{p}=e^{-j(2 \pi / K) \tau_{p}}, \quad b_{p}=e^{-j\left(2 \pi d_{R} / \lambda\right) \cos \phi_{p}}, \\
& c_{p}=e^{-j\left(2 \pi d_{T} / \lambda\right) \cos \theta_{p}}, \quad d_{p}=e^{-j(2 \pi / N) v_{p}} .
\end{aligned}
$$

We study a joint parameter estimator for the parameters of interest $\left\{a_{p}, b_{p}, c_{p}, d_{p}\right\}_{p=1}^{P}$, where $\left|a_{p}\right|=\left|b_{p}\right|=\left|c_{p}\right|=$ $\left|d_{p}\right|=1$, and $w_{p}(i)$ is considered as an unknown nuisance parameter.

Numerous parametric and nonparametric estimation methods have been proposed for the one-dimensional exponential retrieval problem. Only few of these techniques allow a simple extension of the multidimensional case at a reasonable computational load [3]. Simple application of 1D results separately in each dimension is only suboptimal in the sense that it does not exploit the benefits inherent in the multidimensional structure, leading to difficulties in mutually associating the parameter estimates obtained in the various dimensions and over-strict uniqueness conditions [4]. Contrariwise, many parametric high-resolution methods specifically designed for multidimensional frequency estimation require nonlinear and nonconvex optimization so that the computational cost associated with the optimization procedure becomes prohibitively high.

In this paper a novel eigenspace-based estimation method for multidimensional harmonic retrieval problems is proposed. The method can be viewed as an extension to the rank reduction estimator (RARE) [5], originally developed for DOA estimation in partly calibrated arrays. The method is computationally efficient due to its rooting-based implementation, makes explicit use of the rich Vandermonde structure in the measurement data, and therefore shows improved estimation performance compared to conventional search-free methods for multidimensional frequency estimation.
The multidimensional RARE (MD RARE) algorithm estimates the frequencies in the various dimensions sequentially. The dimensionality of the estimation problem and the computational complexity of the estimator is significantly reduced exploiting the Vandermonde structure of the data model. This approach yields high estimation accuracy, moderate identifiability conditions, and automatically associated parameter estimates along the various dimensions. The performance of the algorithm is illustrated at the example of MIMO communication channel estimation based on the double-directional channel model. Numerical examples based on simulated and measured data recorded from the RUSK vector channel sounder at $2 \mathrm{GHz}$ are presented.

\section{SIGNAL MODEL}

For simplicity of notation, we formulate the signal model for the 2D case in detail. Here, the original MIMO channel estimation problem reduces to a single-input multipleoutput (SIMO) channel problem, where the parameters of interest are the propagation delays $\tau_{p}$ and the DOAs $\phi_{p}$ for $p=1, \ldots, P$. Extensions of the proposed algorithm to higher numbers of dimensions are straightforward. Consider a superposition of $P$ discrete-time 2D exponentials corrupted by noise and let $\left(a_{p}, b_{p}\right) \in \mathbb{C}^{1 \times 2},\left|a_{p}\right|=\left|b_{p}\right|=1$, denote the generator pair corresponding to the $p$ th discrete $2 \mathrm{D}$ harmonic,

$$
\begin{gathered}
y_{k, \ell}(i)=\sum_{p=1}^{P} w_{p}(i) a_{p}{ }^{k} b_{p}{ }^{\ell}+n_{k, \ell}(i), \\
i=1, \ldots, J, k=1, \ldots, K, \ell=1, \ldots, L .
\end{gathered}
$$

Here, $a_{p}=e^{-j(2 \pi / K) \tau_{p}}, b_{p}=e^{-j\left(2 \pi d_{R} / \lambda\right) \cos \phi_{p}}, K$ and $L$ mark the sample support along the $a$ - and the $b$-axis, respectively, and $J$ is the number of SIMO snapshots available. The Khatri-Rao product (columnwise Kronecker product) of matrix $\mathbf{U}$ and matrix $\mathbf{V}$ is defined as, $\mathbf{U} \circ \mathbf{V}=\left[\mathbf{u}_{1} \otimes \mathbf{v}_{1}, \mathbf{u}_{2} \otimes \mathbf{v}_{2}, \ldots\right]$, where $\mathbf{u}_{k} \otimes \mathbf{v}_{k}$ is the Kronecker matrix product of the $k$ th column $\mathbf{u}_{k}$ of $\mathbf{U}$ and the $k$ th column $\mathbf{v}_{k}$ of $\mathbf{V}$. Introducing the vector $\boldsymbol{\Omega}=\left[\left(a_{1}, b_{1}\right), \ldots,\left(a_{P}, b_{P}\right)\right]$ containing the parameters of interest, and defining the Vandermonde matrices $[\mathbf{A}]_{i, j}=\left(a_{j}\right)^{i}$, $\mathbf{A} \in \mathbb{C}^{K \times P}$, and $[\mathbf{B}]_{i, j}=\left(b_{j}\right)^{i}, \mathbf{B} \in \mathbb{C}^{L \times P}$, the $2 \mathrm{D}$ harmonic retrieval problem can be stated as follows. Given the measurement data $\mathbf{y}(i)=\left[y_{1,1}(i), y_{2,1}(i), \ldots, y_{K-1, L}(i), y_{K, L}(i)\right]^{T} \in$ $\mathbb{C}^{K L \times 1}$,

$$
\mathbf{y}(i)=\mathbf{H}(\boldsymbol{\Omega}) \mathbf{w}(i)+\mathbf{n}(i), \quad i=1, \ldots, N,
$$

determine the parameter vector $\Omega$ associated with all 2D harmonics. Here, the $2 \mathrm{D}$ signal matrix $\mathbf{H}(\boldsymbol{\Omega})$ is formed as the Khatri-Rao product of the Vandermonde matrices $\mathbf{B}$ and $\mathbf{A}$, that is,

$$
\mathbf{H}(\boldsymbol{\Omega})=\mathbf{B} \circ \mathbf{A} \in \mathbb{C}^{K L \times P},
$$

$\mathbf{y}(i)$ denotes the measurement vector, $\mathbf{w}(i)=\left[w_{1}, \ldots, w_{P}\right]^{T} \in$ $\mathbb{C}^{P \times 1}$ stands for the complex envelope of the $P$ harmonics, 
$\mathbf{n}(i)$ is the vector of additive zero-mean complex (circular) Gaussian noise with covariance matrix $\mathrm{E}\left\{\mathbf{n}(i) \mathbf{n}^{H}(i)\right\}=$ $\sigma^{2} \mathbf{I}_{K L}$. In this paper the linear parameters $\mathbf{w}(i)$ and the noise variance $\sigma^{2}$ are treated as nuisance parameters. Once the parameter vector $\boldsymbol{\Omega}$ is determined the estimation of these parameters is straightforward [6]. Equation (6) describes the 2D harmonic retrieval problem which can be solved by the conventional ESPRIT algorithm [7] and the multidimensional ESPRIT (MD ESPRIT) algorithm [3]. In the following we derive a new search-free eigenspace-based estimation method for the general case in (6) which yields highly accurate estimates of the parameters of interest.

Let the data covariance matrix be given by

$$
\mathbf{R}=\mathrm{E}\left\{\mathbf{y}(i) \mathbf{y}^{H}(i)\right\}=\mathbf{E}_{S} \boldsymbol{\Lambda}_{S} \mathbf{E}_{S}^{H}+\mathbf{E}_{N} \boldsymbol{\Lambda}_{N} \mathbf{E}_{N}^{H},
$$

where $(\cdot)^{H}$ denotes the Hermitian transpose, and $\mathrm{E}\{\cdot\}$ stands for statistical expectation. The diagonal matrices $\boldsymbol{\Lambda}_{S} \in$ $\mathbb{R}^{(P \times P)}$ and $\boldsymbol{\Lambda}_{N} \in \mathbb{R}^{(K L-P) \times(K L-P)}$ contain the signal-subspace and the noise-subspace eigenvalues of $\mathbf{R}$, respectively. In turn, the columns of the matrices $\mathbf{E}_{S} \in \mathbb{C}^{(K L \times P)}$ and $\mathbf{E}_{N} \in$ $\mathbb{C}^{K L \times(K L-P)}$ denote the corresponding signal-subspace and noise-subspace eigenvectors. The finite sample estimates are given by

$$
\hat{\mathbf{R}}=\frac{1}{J} \sum_{i=1}^{J} \mathbf{y}(i) \mathbf{y}^{H}(i)=\hat{\mathbf{E}}_{S} \hat{\boldsymbol{\Lambda}}_{S} \hat{\mathbf{E}}_{S}^{H}+\hat{\mathbf{E}}_{N} \hat{\boldsymbol{\Lambda}}_{N} \hat{\mathbf{E}}_{N}^{H} .
$$

Definition 1. We define the two Vandermonde vectors $\mathbf{a}=$ $\left(1, a, a^{2}, \ldots, a^{K-1}\right)^{T}$ and $\mathbf{b}=\left(1, b, b^{2}, \ldots, b^{L-1}\right)^{T}$. Let $\mathbf{I}_{n}$ be the $n \times n$ identity matrix. We define two "tall" matrices $\mathbf{T}_{a}$ and $\mathbf{T}_{b}$ via

$$
\begin{aligned}
\mathbf{T}_{a}=\mathbf{I}_{L} \otimes \mathbf{a} & \in \mathbb{C}^{K L \times L}, \\
\mathbf{T}_{b} & =\mathbf{b} \otimes \mathbf{I}_{K} \in \mathbb{C}^{K L \times K} .
\end{aligned}
$$

\section{THE 2D RARE ALGORITHM}

In the derivation of the 2D RARE algorithm, we use the following assumptions.

Assumption 1. The number of harmonics does not exceed the smaller of the two numbers $(K-1) L$ and $K(L-1)$, that is,

$$
P \leq K L-\max \{K, L\}
$$

Assumption 2. The signal matrix $\mathbf{H}(\boldsymbol{\Omega}) \in \mathbb{C}^{K L \times P}$ (7) has full column rank $P$.

Assumption 3. The column-reduced signal matrices

$$
\begin{aligned}
& \mathbf{H}_{a}(\boldsymbol{\Omega})=\left[\mathbf{h}_{a, 1}, \ldots, \mathbf{h}_{a, P}\right]=\left(\mathbf{B} \circ \mathbf{A}_{r}\right) \in \mathbb{C}^{(K-1) L \times P}, \\
& \mathbf{H}_{b}(\boldsymbol{\Omega})=\left[\mathbf{h}_{b, 1}, \ldots, \mathbf{h}_{b, P}\right]=\left(\mathbf{B}_{r} \circ \mathbf{A}\right) \in \mathbb{C}^{K(L-1) \times P}
\end{aligned}
$$

with Vandermonde matrices

$$
\begin{aligned}
{\left[\mathbf{A}_{r}\right]_{i, j} } & =\left(a_{j}\right)^{i} \in \mathbb{C}^{(K-1) \times P}, \\
{\left[\mathbf{B}_{r}\right]_{i, j} } & =\left(b_{j}\right)^{i} \in \mathbb{C}^{(L-1) \times P}
\end{aligned}
$$

have full column rank. Note that the matrices $\mathbf{A}_{r}, \mathbf{B}_{r}$ can be obtained from A, B by deleting the last row.

Remark 1. In most realistic applications, Assumptions 2 and 3 hold true almost surely, that is, with probability 1 . Specifically, it can be shown that if the generators $\left\{\left(a_{p}, b_{p}\right)\right\}_{p=1}^{P}$ are drawn from a distribution $P_{\mathcal{L}}\left(\mathbb{C}^{2 P}\right)$ that is assumed to be continuous with respect to the Lebesgue measure in $\mathbb{C}^{2 P}$, then the violation of Assumptions 2 and 3 is a probabilityzero event [4].

Remark 2. Note that Assumption 3 implies that each generator $a_{i}$ and $b_{j}$ occurs with multiplicity $M_{a}<L$ and $M_{b}<K$ in the generator sets $\left\{a_{i}\right\}_{i=1}^{P}$ and $\left\{b_{j}\right\}_{j=1}^{P}$, respectively.

See Appendix A for the proof.

Proposition 1. Provided that Assumptions 1, 2, and 3 are satisfied, the augmented matrix

$$
\mathbf{G}_{a}=\left[\mathbf{T}_{a} \mid \mathbf{H}(\mathbf{\Omega})\right] \in \mathbb{C}^{K L \times(L+P)}
$$

has full column rank if and only if $a \neq a_{p}$, for $p=1, \ldots, P$, $P \leq L(K-1)$. Similarly, provided that Assumptions 1, 2 and 3 are satisfied, the augmented matrix

$$
\mathbf{G}_{b}=\left[\mathbf{T}_{b} \mid \mathbf{H}(\mathbf{\Omega})\right] \in \mathbb{C}^{K L \times(K+P)}
$$

has full column rank if and only if $b \neq b_{p}$ for $p=1, \ldots, P$, $P \leq(L-1) K$.

See Appendix B for the proof.

With Proposition 1 and provided that $\left\{a_{1}, \ldots, a_{P}\right\}$ are the true signal generators along the $a$-axis, the quadratic form

$$
\begin{aligned}
F_{R, a}(a) & =\gamma^{H} \mathbf{H}(\boldsymbol{\Omega})^{H}\left(\mathbf{I}_{K L}-\mathbf{T}_{a}\left(\mathbf{T}_{a}^{H} \mathbf{T}_{a}\right)^{-1} \mathbf{T}_{a}^{H}\right) \mathbf{H}(\mathbf{\Omega}) \boldsymbol{\gamma} \\
& =0, \quad \text { for } a \in\left\{a_{1}, \ldots, a_{P}\right\} \\
& >0, \quad \text { otherwise, }
\end{aligned}
$$

for arbitrary vector $\boldsymbol{\gamma} \in \mathbb{C}^{P} \backslash\{\mathbf{0}\},|a|=1$, and $P \leq L(K-1)$. It can readily be verified that the signal matrix $\mathbf{H}(\Omega)$ and the signal-subspace matrix $\mathbf{E}_{S}$ span the same subspace [6], that is, there exist a full-rank matrix $\mathbf{K}$ such that $\mathbf{H}(\boldsymbol{\Omega})=\mathbf{E}_{S} \mathbf{K}$. From identity (16), we can formulate one of the main results of the paper.

Proposition 2. Provided that $\left\{a_{1}, \ldots, a_{P}\right\}$ are the true signal generators along the a-axis, then

$$
\begin{aligned}
F_{R, a}(a) & =\tilde{\gamma}^{H} \mathbf{E}_{S}^{H}\left(\mathbf{I}_{K L}-\mathbf{T}_{a}\left(\mathbf{T}_{a}^{H} \mathbf{T}_{a}\right)^{-1} \mathbf{T}_{a}^{H}\right) \mathbf{E}_{S} \tilde{\boldsymbol{\gamma}} \\
& =0, \quad \text { for } \mathrm{a} \in\left\{\mathrm{a}_{1}, \ldots, \mathrm{a}_{\mathrm{P}}\right\}, \\
& >0, \quad \text { otherwise, }
\end{aligned}
$$

where $\boldsymbol{\gamma} \in \mathbb{C}^{P} \backslash\{\mathbf{0}\}, \tilde{\boldsymbol{\gamma}}=\mathbf{K} \boldsymbol{\gamma},|a|=1, P \leq L(K-1)$, and $\mathbf{K}$ is defined as above. 
Since $\tilde{\gamma}$ is an arbitrary nonzero vector, identity (17) easily translates into an equivalent condition on the harmonic $a$ given by

$$
\begin{aligned}
F_{R, a}(a) & =\operatorname{det}\left\{\mathbf{E}_{S}^{H}\left(\mathbf{I}_{K L}-\mathbf{T}_{a}\left(\mathbf{T}_{a}^{H} \mathbf{T}_{a}\right)^{-1} \mathbf{T}_{a}^{H}\right) \mathbf{E}_{S}\right\} \\
& =0, \quad \text { for } a \in\left\{a_{1}, \ldots, a_{P}\right\} \\
& >0, \quad \text { otherwise. }
\end{aligned}
$$

In other words, the $1 \mathrm{D}$ matrix polynomial

$$
\mathbf{M}_{a}(a) \triangleq \mathbf{E}_{S}^{H}\left(\mathbf{I}_{K L}-\mathbf{T}_{a}\left(\mathbf{T}_{a}^{H} \mathbf{T}_{a}\right)^{-1} \mathbf{T}_{a}^{H}\right) \mathbf{E}_{S} \in \mathbb{C}^{P \times P}
$$

becomes singular (i.e., rank deficient) at exactly $P$ locations $a$ with $|a|=1$. These locations $a$ are the true generators $\left\{a_{p}\right\}_{p=1}^{P}$. In accordance with (18), the fundamental idea of the 2D RARE algorithm consists in determining the $P$ true harmonics from the roots of the RARE matrix polynomial $\mathbf{M}_{a}(a)$ located on the unit circle, that is, the true generators $\left\{a_{p}\right\}_{p=1}^{P}$ are given by the solutions of the polynomial equation

$$
\begin{aligned}
\left.F_{R, a}(a)\right|_{|a|=1} & =\operatorname{det}\left\{\mathbf{E}_{S}^{H}\left(\mathbf{I}_{K L}-\mathbf{T}_{a}\left(\mathbf{T}_{a}^{H} \mathbf{T}_{a}\right)^{-1} \mathbf{T}_{a}^{H}\right) \mathbf{E}_{S}\right\} \\
& =0 .
\end{aligned}
$$

Up to now we have considered estimating the generator $a$ along a single data dimension, that is, the $a$-axis. The solution of (20) corresponds to the 1D RARE algorithm for harmonic retrieval originally proposed in [5]. Following similar consideration as above, Proposition 1 reveals that the true generators $\left\{b_{p}\right\}_{p=1}^{P}$ are given by the roots of the $1 \mathrm{D}$ matrix polynomial in $b$,

$$
\mathbf{M}_{b}(b) \triangleq \mathbf{E}_{S}^{H}\left(\mathbf{I}_{K L}-\mathbf{T}_{b}\left(\mathbf{T}_{b}^{H} \mathbf{T}_{b}\right)^{-1} \mathbf{T}_{b}^{H}\right) \mathbf{E}_{S} \in \mathbb{C}^{P \times P},
$$

evaluated on the unit circle. The associated RARE polynomial equation reads

$$
\begin{aligned}
\left.F_{R, b}(b)\right|_{|b|=1} & =\operatorname{det}\left\{\mathbf{E}_{S}^{H}\left(\mathbf{I}_{K L}-\mathbf{T}_{b}\left(\mathbf{T}_{b}^{H} \mathbf{T}_{b}\right)^{-1} \mathbf{T}_{b}^{H}\right) \mathbf{E}_{S}\right\} \\
& =0 .
\end{aligned}
$$

In the finite sample case, the true signal-subspace eigenvectors $\mathbf{E}_{S}$ in (20) and (22) are replaced by their finite sample estimates defined in (9). Due to finite sample and noise effects, the signal roots of the RARE polynomial equations are displaced from their ideal positions on the unit circle. In this case the signal roots are determined as the $P$ roots of (20) and (22) inside the unit circle that are located closest to the unit circle [8].

In the preceding considerations, the estimation criteria provided by (20) and (22) were derived from Proposition 1 to separately determine the generator sets $\left\{a_{p}\right\}_{p=1}^{P}$ and $\left\{b_{p}\right\}_{p=1}^{P}$. Interestingly, Proposition 1 can further be exploited to develop a parameter association procedure from which the true parameter pairs $\left\{\left(a_{p}, b_{p}\right)\right\}_{p=1}^{P}$ are efficiently obtained.
Corollary 1. Given the true generator sets $\left\{a_{p}\right\}_{p=1}^{P}$ and $\left\{b_{p}\right\}_{p=1}^{P}$, we construct the $2 D$ matrix polynomial via the convex linear combination of (19) and (21),

$$
\overline{\mathbf{M}}(a, b)=\alpha \mathbf{M}_{a}(a)+(1-\alpha) \mathbf{M}_{b}(b) .
$$

This $2 D$ matrix polynomial becomes singular for real $0<\alpha<$ 1 if and only if $(a, b)$ is a true generator pair. Specifically, if $\left(a_{p}, b_{p}\right)$ denotes the generator pair of the pth harmonic, then $\overline{\mathbf{M}}\left(a_{p}, b_{p}\right)$ contains exactly one zero eigenvalue $\left(\rho_{p, 0}=0\right)$ with the associated eigenvector $\tilde{\gamma}_{p, 0}=\mathbf{k}_{p}$ denoting the pth column of the full-rank matrix $\mathbf{K}$ defined through relation $\mathbf{H}(\boldsymbol{\Omega})=\mathbf{E}_{S} \mathbf{K}$, here equivalence holds up to complex scaling of the columns of K.

See Appendix C for the proof.

Corollary 1 provides a powerful tool for associating the two sets of parameter estimates $\left\{\hat{a}_{i}\right\}_{i=1}^{P}$ and $\left\{\hat{b}_{j}\right\}_{j=1}^{P}$ that were separately obtained from the RARE criteria (20) and (22) along the $a$ - and the $b$-axis, respectively. For a specific harmonic $\hat{a}_{i}$ of the first set, the corresponding harmonic $\hat{b}_{j}$ of the second set is given by the element of $\left\{\hat{b}_{j}\right\}_{j=1}^{P}$ that minimizes the cost function

$$
\begin{aligned}
F_{\text {pair }, i}(j) & =\lambda_{\min }\left\{\overline{\mathbf{M}}\left(\hat{a}_{i}, \hat{b}_{j}\right)\right\} \\
& =\lambda_{\min }\left\{\alpha \mathbf{M}\left(\hat{a}_{i}\right)+(1-\alpha) \mathbf{M}\left(\hat{b}_{j}\right)\right\}
\end{aligned}
$$

for an appropriately chosen $\alpha$ between 0 and 1 . Here, $\lambda_{\min }\left\{\overline{\mathbf{M}}\left(\hat{a}_{i}, \hat{b}_{j}\right)\right\}$ denotes the smallest eigenvalue of $\overline{\mathbf{M}}\left(\hat{a}_{i}, \hat{b}_{j}\right)$ (23).

\section{IMPLEMENTATION}

In this section we provide a short description of the $4 \mathrm{D}$ RARE algorithm for estimating the $4 \mathrm{D}$ harmonics associated with the general channel estimation problem in (3) for the finite sample case. Define the generator sets $\Phi_{1}=\left\{a_{1}, \ldots, a_{P}\right\}$, $\Phi_{2}=\left\{b_{1}, \ldots, b_{P}\right\}, \Phi_{3}=\left\{c_{1}, \ldots, c_{P}\right\}$, and $\Phi_{4}=\left\{d_{1}, \ldots, d_{P}\right\}$ and initialize source index $S=0$.

Step 1. Estimate the sample covariance matrix $\hat{\mathbf{R}}$ and the signal-subspace eigenvectors $\hat{\mathbf{E}}_{S}$, for example, from (9).

Step 2. Find the roots of the RARE polynomials along the four dimensions

$$
\begin{aligned}
& F_{R, a}(a)=\operatorname{det}\left\{\hat{\mathbf{E}}_{S}^{H}\left(\mathbf{I}-\mathbf{T}_{a}\left(\mathbf{T}_{a}^{H} \mathbf{T}_{a}\right)^{-1} \mathbf{T}_{a}^{H}\right) \hat{\mathbf{E}}_{S}\right\}=0, \\
& F_{R, b}(b)=\operatorname{det}\left\{\hat{\mathbf{E}}_{S}^{H}\left(\mathbf{I}-\mathbf{T}_{b}\left(\mathbf{T}_{b}^{H} \mathbf{T}_{b}\right)^{-1} \mathbf{T}_{b}^{H}\right) \hat{\mathbf{E}}_{S}\right\}=0, \\
& F_{R, c}(c)=\operatorname{det}\left\{\hat{\mathbf{E}}_{S}^{H}\left(\mathbf{I}-\mathbf{T}_{c}\left(\mathbf{T}_{c}^{H} \mathbf{T}_{c}\right)^{-1} \mathbf{T}_{c}^{H}\right) \hat{\mathbf{E}}_{S}\right\}=0, \\
& F_{R, d}(d)=\operatorname{det}\left\{\hat{\mathbf{E}}_{S}^{H}\left(\mathbf{I}-\mathbf{T}_{d}\left(\mathbf{T}_{d}^{H} \mathbf{T}_{d}\right)^{-1} \mathbf{T}_{d}^{H}\right) \hat{\mathbf{E}}_{S}\right\}=0
\end{aligned}
$$


for

$$
\begin{aligned}
& \mathbf{T}_{a}=\mathbf{I}_{L M N} \otimes \mathbf{a} \in \mathbb{C}^{K L M N \times L M N}, \\
& \mathbf{T}_{b}=\mathbf{I}_{M N} \otimes \mathbf{b} \otimes \mathbf{I}_{K} \in \mathbb{C}^{K L M N \times M N K}, \\
& \mathbf{T}_{c}=\mathbf{I}_{N} \otimes \mathbf{c} \otimes \mathbf{I}_{K L} \in \mathbb{C}^{K L M N \times N K L,} \\
& \mathbf{T}_{d}=\mathbf{d} \otimes \mathbf{I}_{K L M} \in \mathbb{C}^{K L M N \times K L M},
\end{aligned}
$$

and we substitute $\mathbf{T}_{a}^{H} \triangleq \mathbf{T}_{1 / a}^{T}, \mathbf{T}_{b}^{H} \triangleq \mathbf{T}_{1 / b}^{T}, \mathbf{T}_{c}^{H} \triangleq \mathbf{T}_{1 / c}^{T}$, and $\mathbf{T}_{d}^{H} \triangleq \mathbf{T}_{1 / d}^{T}$.

Step 3. Determine estimates of the generator sets $\Phi_{1}, \Phi_{2}, \Phi_{3}$, and $\Phi_{4}$ as the roots located closest to the unit circle of the polynomials in (25) and denoted by $\Phi_{1}^{(P)}, \Phi_{2}^{(P)}, \Phi_{3}^{(P)}$ and $\Phi_{4}^{(P)}$, respectively. Here, the superscript " $(P)$ " indicates the number of elements in the set. Let $\hat{u}_{i, k}$ denote the $k$ th element of the $i$ th set.

Step 4 . Set $S:=S+1$. Pick a well-separated ${ }^{1}$ generator $\hat{u}_{i, k}$ from any of the sets $\left\{\Phi_{i}^{(P-S+1)}\right\}$ for $i=1, \ldots, 4$.

Step 5. For $j=1, \ldots, 4$ with $j \neq i$, find the corresponding root $\hat{u}_{j, m}$ from the set $\Phi_{j}^{(P-S+1)}$ such that the cost function

$$
\begin{aligned}
F_{\text {pair }, k}^{(i, j)}(m) & =\lambda_{\min }\left\{\overline{\mathbf{M}}\left(\hat{u}_{i, k}, \hat{u}_{j, m}\right)\right\} \\
& =\lambda_{\min }\left\{\alpha \mathbf{M}\left(\hat{u}_{i, k}\right)+(1-\alpha) \mathbf{M}\left(\hat{u}_{j, m}\right)\right\}
\end{aligned}
$$

is minimized for fixed $\alpha$ between 0 and 1 . Store the solution $\hat{u}_{j, m}$ in the $(j, S)$ th entry of the $(4 \times P)$ association matrix $\hat{\mathbf{Z}}$ and remove it from the set $\Phi_{j}^{(P-S+1)}$.

Step 6. Repeat Steps 4 and 5 until $S=P$ and all entries of the $(4 \times P)$ association matrix $\hat{\mathbf{Z}}$ are filled. Matrix $\hat{\mathbf{Z}}$ represents the RARE estimate of the true generator matrix $\mathbf{Z}$,

$$
\mathbf{Z}=\left[\begin{array}{llll}
a_{1} & a_{2} & \cdots & a_{P} \\
b_{1} & b_{2} & \cdots & b_{P} \\
c_{1} & c_{2} & \cdots & c_{P} \\
d_{1} & d_{2} & \cdots & d_{P}
\end{array}\right]
$$

with mutually associated harmonic estimates along its columns.

Step 7. For each $4 \mathrm{D}$ harmonic $\left(\hat{a}_{i}, \hat{b}_{i}, \hat{c}_{i}, \hat{d}_{i}\right), i=1, \ldots, P$, obtained in the previous step, determine the corresponding delay $\hat{\tau}_{i}$, the direction of arrival $\hat{\phi}_{i}$, the DOD $\hat{\theta}_{i}$, and the Doppler shift $\hat{v}_{i}$ according to the arguments of the estimates in (4).

The source parameter association procedure in Steps 5, 6, and 7 is based on the pairwise association of all $4 \mathrm{D}$ harmonics and stems from the observation that all $4 \mathrm{D}$ harmonics are

\footnotetext{
${ }^{1}$ In order to guarantee uniqueness and best performance in the parameter association, it is recommended to pick a root $\hat{u}_{i, k}$ (and an associated set $\Phi_{i}^{(P-S+1)}$ ) which is well separated in terms of angular distance $d_{i}(k, l)=\left|\arg \left\{\hat{u}_{i, k}\right\}-\arg \left\{\hat{u}_{i, l}\right\}\right|$ from the remaining roots $\left\{\hat{u}_{i, l}\right\}_{l \neq k, l=1}^{(P-S+1)}$ in the set.
}

TABLE 1: Generators of the 3D harmonics used for simulation with synthetic data in Section 5.1.

\begin{tabular}{c|ccc}
\hline$P=3$ & $a_{p}$ & $b_{p}$ & $c_{p}$ \\
\hline$p=1$ & $e^{j 0.550 \pi}$ & $e^{j 0.719 \pi}$ & $e^{j 0.906 \pi}$ \\
$p=2$ & $e^{j 0.410 \pi}$ & $e^{j 0.777 \pi}$ & $e^{j 0.276 \pi}$ \\
$p=3$ & $e^{j 0.340 \pi}$ & $e^{j 0.906 \pi}$ & $e^{j 0.358 \pi}$ \\
\hline
\end{tabular}

separated in at least one dimension. With Corollary 1 , this observation facilitates the parameter association in the sense that the general $4 \mathrm{D}$ parameter association problem can be reduced to the much simpler pairing problem of multiple $2 \mathrm{D}$ harmonics.

\section{NUMERICAL RESULTS}

\subsection{Simulation with synthetic data}

In this section simulation results using synthetic data are presented. Computer simulations are carried out for the $3 \mathrm{D}$ case. The signal model is defined in (3), but without the harmonics $d_{p}$ and the last dimension $n$ collapses to a singleton $n=1$. The sample sizes along the $a-, b$-, and $c$-axes are chosen as $K=L=M=5$ and the $\mathbf{y}(i)$ vectors have dimension $5^{3}$. The $\left(5^{3} \times 5^{3}\right)$ data covariance matrix is computed from $J=10$ independent snapshots and a number of $P=3$ equi-powered exponentials is assumed with the generators $\boldsymbol{\Omega}=\operatorname{vec}\{\mathbf{Z}\}=\left[\left(a_{1}, b_{1}, c_{1}\right),\left(a_{2}, b_{2}, c_{2}\right),\left(a_{3}, b_{3}, c_{3}\right)\right]^{T}$ given in Table 1. Forward-backward averaging is used to increase the effective snapshot number in order to obtain from (9) a covariance matrix estimate of sufficiently high rank. The simulations are carried out according to the signal model (6) with complex Gaussian $\mathbf{c}(i)$, zero mean, with covariance $\mathrm{E}\left\{\mathbf{c}(i) \mathbf{c}^{H}(i)\right\}=\mathbf{I}_{3}$ and $\mathrm{E}\left\{\mathbf{c}(i) \mathbf{c}^{T}(i)\right\}=\mathbf{0}$. Complex zero-mean Gaussian noise $\mathbf{n}(i)$ is added according to (6) with covariance matrix $\mathrm{E}\left\{\mathbf{n}(i) \mathbf{n}^{H}(i)\right\}=\sigma^{2} \mathbf{I}_{125}$ and $\mathrm{E}\left\{\mathbf{n}(i) \mathbf{n}^{T}(i)\right\}=\mathbf{0}$. The root mean squared error (RMSE) of the parameter estimates obtained by the multidimensional RARE algorithm averaged over $R=100$ simulation runs are plotted versus the signal to noise ratio (SNR) in Figure 1. We used the following definitions:

$$
\begin{gathered}
\operatorname{SNR}=\frac{1}{\sigma^{2}}, \\
\operatorname{RMSE}(a)=\left(\frac{1}{R P} \sum_{r=1}^{R} \sum_{p=1}^{P}\left|\arg \left(\left(\hat{a}_{p}\right)_{r}\right)-\arg \left(a_{p}\right)\right|^{2}\right)^{1 / 2},
\end{gathered}
$$

where $\left(\hat{a}_{p}\right)_{r}$ denotes the estimate for $a_{p}$ obtained in the $r$ th simulation run (and similarly for the $b$-and $c$-generators). A comparison to the corresponding Cramer-Rao bound (CRB) [9] and to results obtained from the unitary ESPRIT algorithm [3] reveals that the new method yields estimation performance close to the CRB and clearly outperforms the popular unitary ESPRIT estimator which is based on the joint Schur decomposition.

In Figure 2 we investigate the effect of the weighting parameter $\alpha$ used in Step 5 on the parameter association 


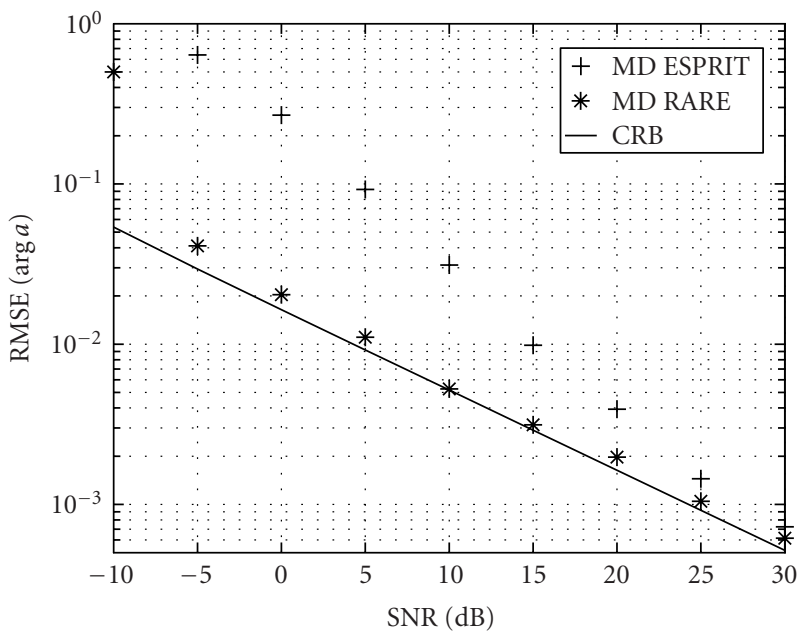

(a)

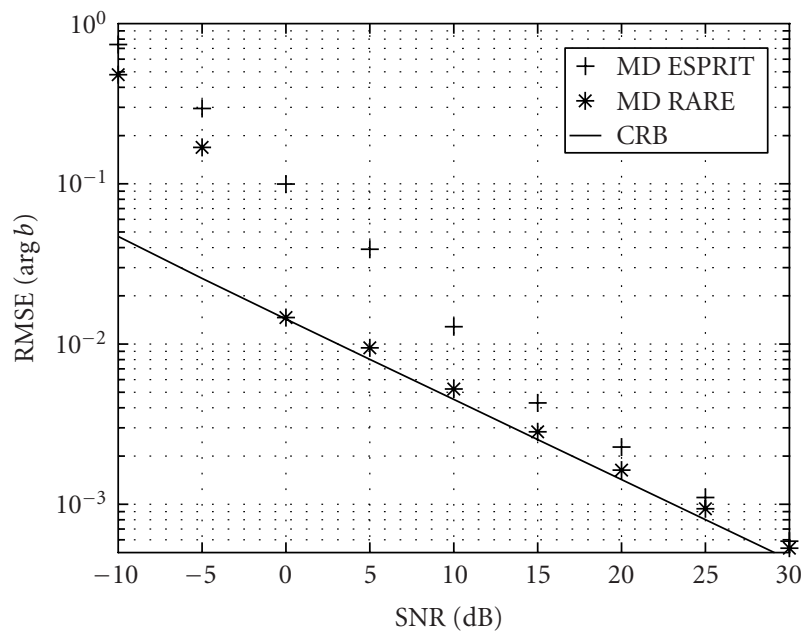

(b)

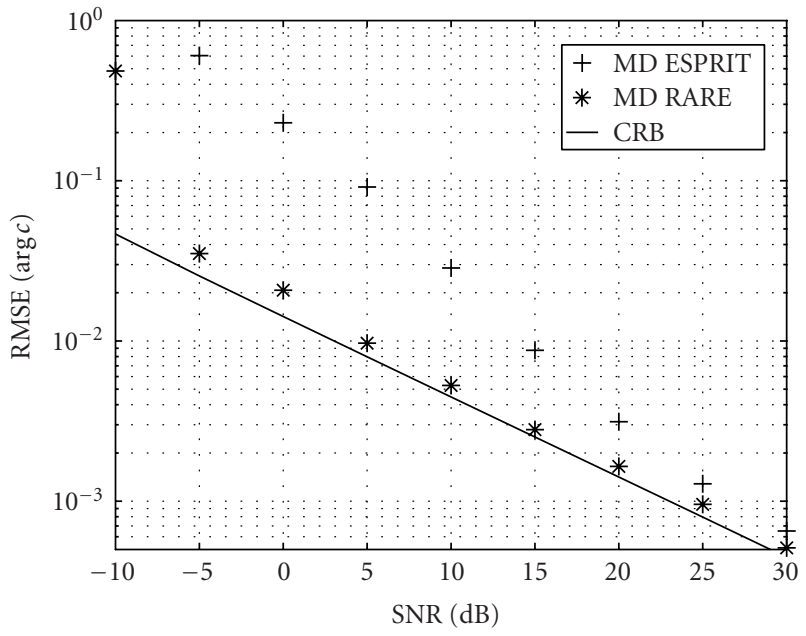

(c)

FIGURE 1: Root mean squared error of 3D RARE versus SNR.

performance. For this purpose we sorted the estimates $\left\{\left(\arg \hat{a}_{i}, \arg \hat{b}_{i}, \arg \hat{c}_{i}\right)\right\}_{i=1}^{3}$ obtained by the 3D RARE algorithm according to $\left\{\arg \hat{a}_{i}\right\}_{i=1}^{3}$ and plotted the RMSE of the estimates $\left\{\arg \hat{b}_{i}\right\}_{i=1}^{3}$ and $\left\{\arg \hat{c}_{i}\right\}_{i=1}^{3}$ against the choice of $\alpha$ for the SNR values $-5,0,5$, and $10 \mathrm{~dB}$. From the simulations, we observe that the proposed parameter association procedure is robust against the choice of $\alpha$ and performs well for a wide range of $\alpha$ taken around the intuitively expected uniform weighting factor $\alpha=0.5$. We observe that a particular choice of $\alpha$ may only affect the performance of the parameter association procedure close to threshold domain while asymptotically the choice of the weighting factor becomes less crucial.

\subsection{Measurement data}

Measurement data were recorded with the RUSK-ATM vector channel sounder, manufactured and marketed by MEDAV [2]. The measurement data used for the numerical experiments in this paper were recorded during a measurement run in Weikendorf, a suburban area in a small town ap- proximately $50 \mathrm{~km}$ north of Vienna, Austria, in autumn 2001 $[10,11]$. The measurement area covers one-family houses with private gardens around them. The houses are typically one floor high. A rail-road track is present in the environment which breaks the structure of single placed houses. A small pedestrian tunnel passes below the railway. A map of the environment with the position of the receiver and transmitter is shown in Figure 3.

The sounder operated at a center frequency of $2000 \mathrm{MHz}$ with an output power of 2 Watt and a transmitted signal bandwidth of $120 \mathrm{MHz}$. The transmitter emitted a periodically repeated signal composed of 384 subcarriers in the band $1940-2060 \mathrm{MHz}$. The repetition period was $3.2 \mathrm{mi}-$ croseconds. The transmitter was the mobile station and the receiver was at a fixed location. The transmit array had a uniform circular geometry composed of 15 monopoles arranged on a ground plane at an interelement spacing of $0.43 \lambda \approx 6.45 \mathrm{~cm}$. The mobile transmitter was mounted on top of a small trolley together with the uniform circular array at a height of approximately $1.5 \mathrm{~m}$ above ground level. At 

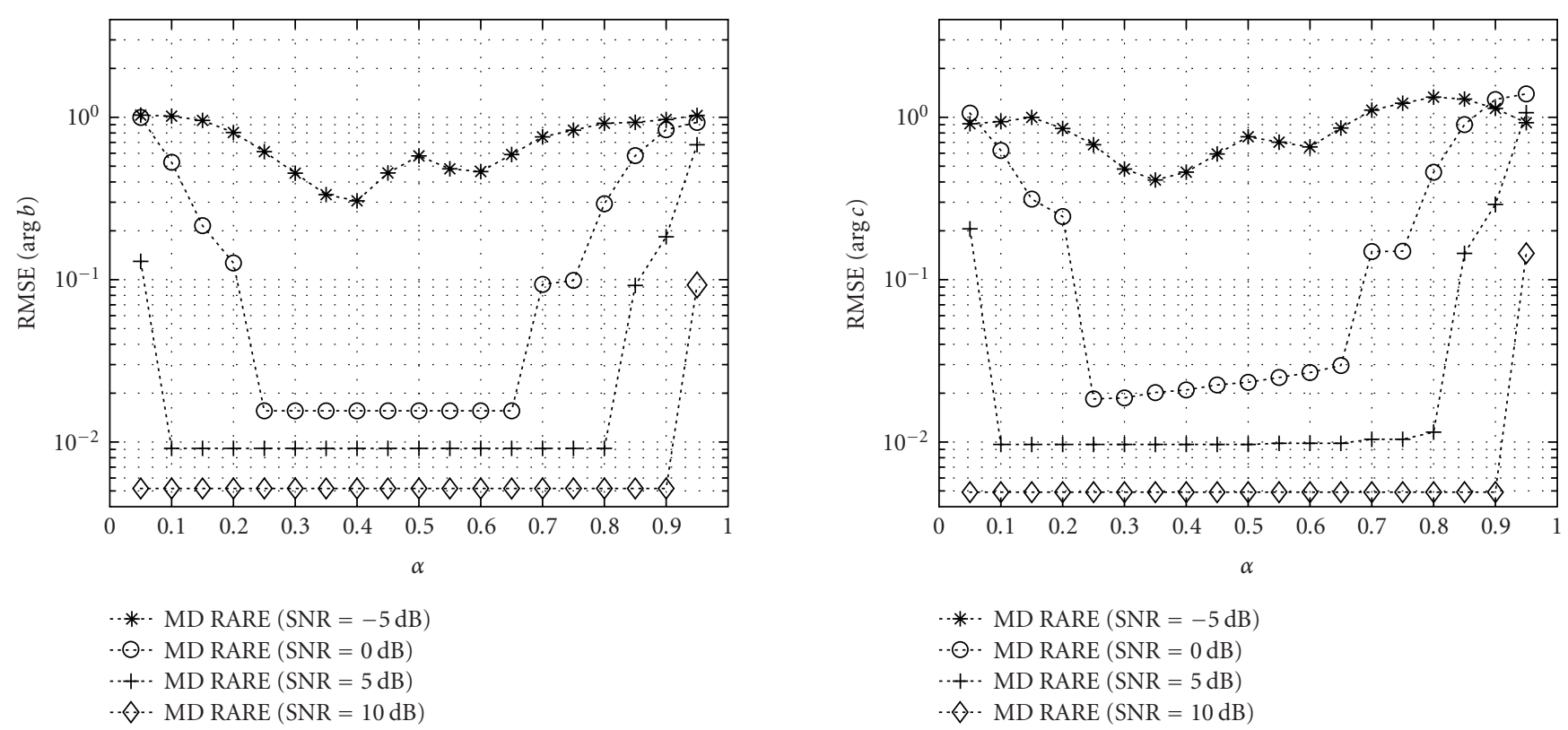

Figure 2: Root mean squared error of 3D RARE versus $\alpha$.

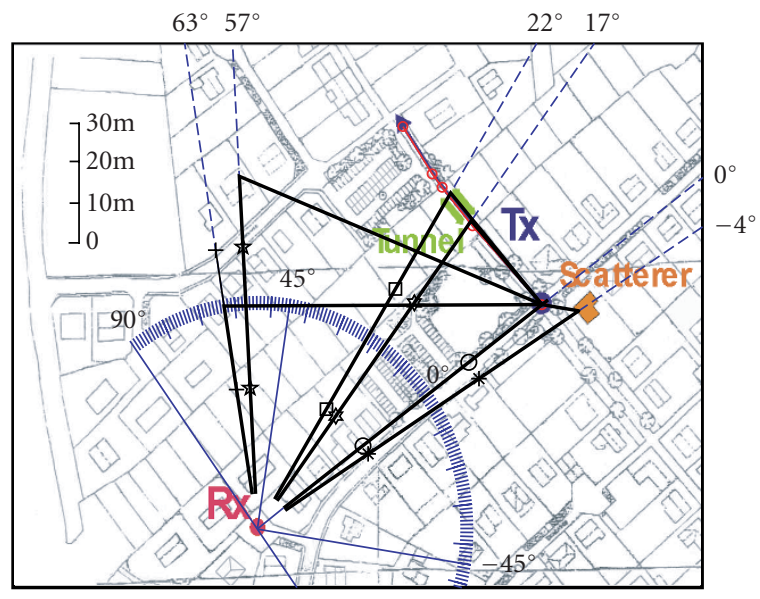

FIGURE 3: Map of the measurement scenario in Weikendorf.

the receiver site, a uniform linear array ${ }^{2}$ composed of 8 elements with half wavelength distance $(7.5 \mathrm{~cm})$ between adjacent patch-elements was mounted on a lift in approximately 20 m height.

With this experimental arrangement, consecutive sets of the $(15 \times 8)$ individual transfer functions, cross-multiplexed in time, were acquired. The receiver calculates the discrete Fourier transform over data blocks of duration 3.2 microseconds and deconvolves the data in the frequency domain with the known transmit signal. The effects from mutual coupling between Rx antenna elements are reduced by multiplying the measurement snapshots $\mathbf{y}(i)$ with a complex-symmetric cor-

\footnotetext{
${ }^{2} \mathrm{~A}$ uniform linear array was provided by T-Systems NOVA, Darmstadt, Germany.
}

rection matrix [12]. The acquisition period of 3.2 microseconds corresponds to a maximum path length of approximately $1 \mathrm{~km}$. During the measurements the receiver moved at speeds of approx. $5 \mathrm{~km} / \mathrm{h}$ on the sidewalk. Rx position and Tx position as well as the motion of the transmitter are marked in the site map in Figure 3. The transmitter passed through a pedestrian tunnel approximately between times $t=25 \mathrm{sec}-$ onds and $t=30$ seconds of the measurement run.

We estimated the data covariance matrix from $J=10$ consecutive snapshots in time. The measurement system in this experiment differs from the data acquisition model described in the Introduction (1), (2), (3), and (4) in that a uniform circular array instead of a uniform linear array was used at the transmitter side. Therefore we can not simply apply the estimation procedure for the $4 \mathrm{D}$ parameter estimation problem described in Section 4 to estimate the directions of departure. In this experiment we only consider the 2D model (5) instead of the general 4D model (1), (2), (3), and (4). In specific we are interested in estimating only the directions of arrival and the time delays. In order to still exploit the complete 4D measurement block that was recorded as described above, we use smoothing over frequency bins and averaging over Tx samples in order to increase the number of snapshots and to obtain a full-rank covariance matrix estimate of reduced variance. Due to the smoothing over frequency bins, the original sample support of $K=384$ frequency bins, along the $a$-axis is reduced to a sample support of $K^{\prime}=12$. For further variance reduction we apply forward-backward (FB) averaging [3]. Making use of the notation of the general $4 \mathrm{D}$ model in (3) the smoothed FB sample covariance matrix corresponding to (8) reads

$$
\hat{\mathbf{R}}=\frac{1}{D} \sum_{i=1}^{J} \sum_{k=1}^{K-K^{\prime}} \sum_{m=1}^{M}\left(\tilde{\mathbf{y}}_{k, m}(i) \tilde{\mathbf{y}}_{k, m}^{H}(i)+\tilde{J}_{k, m}^{*}(i) \tilde{\mathbf{y}}_{k, m}^{T}(i) \mathbf{J}\right)
$$




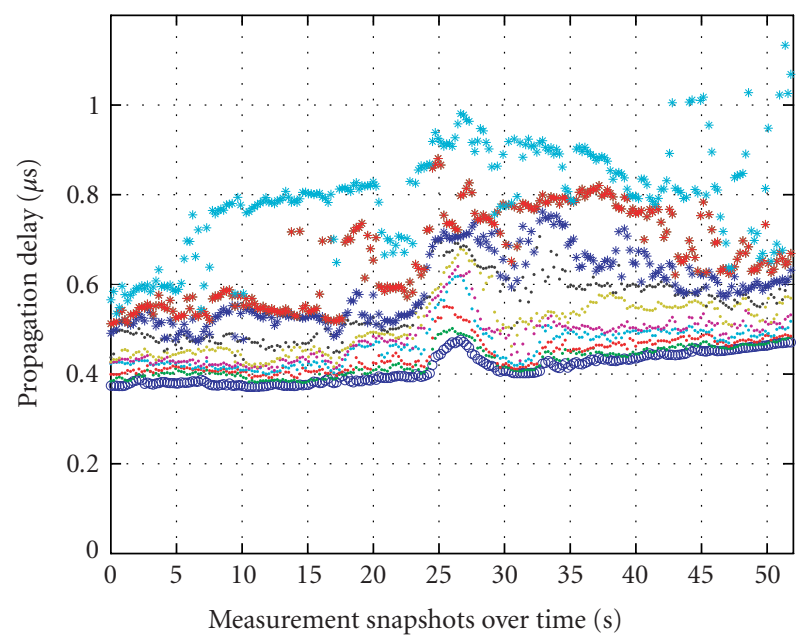

FIgURE 4: Estimates of propagation delay versus snapshots in time.

where $D=J\left(K-K^{\prime}\right) M$,

$$
\begin{array}{r}
\tilde{\mathbf{y}}_{k, m}(i) \\
=\operatorname{vec}\left\{\left[\begin{array}{cccc}
y_{k, 1, m, 1}(i) & y_{k, 2, m, 1}(i) & \cdots & y_{k, L, m, 1}(i) \\
y_{k+1,1, m, 1}(i) & y_{k+1,2, m, 1}(i) & \cdots & y_{k+1, L, m, 1}(i) \\
\vdots & \vdots & \ddots & \vdots \\
y_{k+K^{\prime}, 1, m, 1}(i) & y_{k+K^{\prime}, 2, m, 1}(i) & \cdots & y_{k+K^{\prime}, L, m, 1}(i)
\end{array}\right]\right\},
\end{array}
$$

$M=15, L=8, \mathbf{J}$ denotes the $96 \times 96$ exchange matrix, and $\mathbf{x}=\operatorname{vec}\{\mathbf{X}\}$ denotes the vectorization operator, stacking the columns of a matrix $\mathbf{X}$ on top of each other to form a long vector $\mathbf{x}$. Propagation delay and DOA estimates obtained with 2D RARE are displayed in Figures 4 and 5 relative to the orientation of the array. ${ }^{3}$ We have assumed $P=10$ paths and applied 2D RARE for the joint estimation of propagation delay and DOA. In these two figures, the estimates are plotted as colored marks (“.” and “*”) versus measurement time in seconds. The pairing of the estimates is indicated by the chosen mark and its color. In these figures, the circles ("०") mark the line of sight path, dots (".") mark the consecutive early arrivals whereas the asterisks (“*”) mark the late ones.

We see that the propagation scenario is dominated by a strong line-of-sight (LOS) component surrounded by local scattering paths from trees and buildings during the first 25 seconds of the experiment (shown with the "o" mark in the figures). The trace of the DOA estimates in Figure 5 and the corresponding propagation delay estimates in Figure 4 match exactly the motion of the transmitter depicted in Figure 3 for the direct path. At time 25 seconds, the trolley reaches the pedestrian tunnel and a second path resulting from scattering

\footnotetext{
${ }^{3}$ An animated movie generated from these results can be downloaded from FTW's MIMO measurements, http://www.ftw.at/measurements.
}

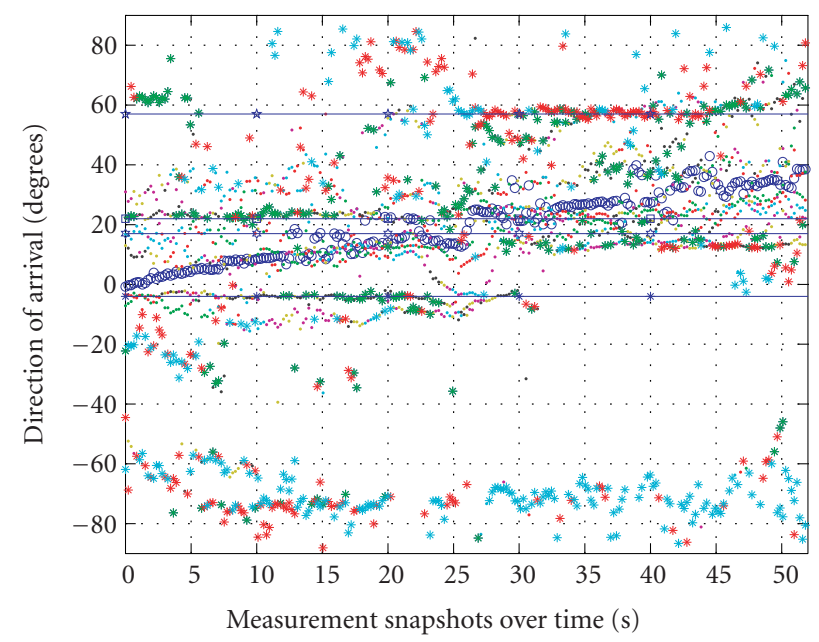

FIGURE 5: Estimates of directions of arrival versus snapshots in time.

at the building (see Figure 5) appears at a DOA of approximately $-3^{\circ}$. This path corresponds to a significantly larger access delay of approx. 0.55-0.58 microseconds. By the time the Tx moves out of the tunnel, the dominant LOS component with local scattering is newly tracked by the 3D RARE algorithm. In Figure 5 we observe a path emerging at a constant DOA of approx. $22^{\circ}$ between snapshot time 0 second and 25 seconds. Similarly, a path emerging at a constant DOA of approx. $17^{\circ}$ between time 28 seconds and $52 \mathrm{sec}-$ onds. These paths are interpreted as contributions from the two ends of the pedestrian tunnel. Furthermore, it is interesting to observe that those propagation paths that show large delay estimates generally yield corresponding DOA estimates with large angular deviations from the line of sight.

\section{SUMMARY AND CONCLUSIONS}

A novel method for $K$-dimensional harmonic exponential estimation has been derived as a multidimensional extension of the conventional RARE algorithm. High-resolution frequency parameter estimates are obtained from the proposed method in a search-free procedure at relatively low computational complexity. The parameters in the various dimensions are independently estimated exploiting the rich structure of the multidimensional measurement model and the estimates of the parameters of interest are automatically associated. Simulation results based on synthetic and measured data of a MIMO communication channel underline the strong performance of the new approach. Finally, we conclude that the double-directional parametric MIMO model (3) is very suitable for describing wireless MIMO channels.

\section{APPENDICES}

\section{A. PROOF OF REMARK 2}

We prove by contradiction that $M_{a}<L$ is necessary for $\mathbf{H}_{b}(\boldsymbol{\Omega})$ to be full rank. Without loss of generality, we assume 
that $P=M_{a}=L$ with $a_{1}=a_{2}=\cdots=a_{L}=a$. In this case we have $\mathbf{H}_{b}(\boldsymbol{\Omega})=\left(\mathbf{B}_{r} \circ \mathbf{A}\right)=\left(\mathbf{T}_{a} \mathbf{B}_{r}\right)$ where $\mathbf{T}_{a}$ is defined according to Definition 1 . Due to the orthogonality of the columns of $\mathbf{T}_{a}$, we have $\operatorname{rank}\left\{\mathbf{T}_{a}\right\}=L$. Applying Sylvester's inequality yields

$$
\begin{aligned}
\operatorname{rank} & \left\{\mathbf{T}_{a}\right\}+\operatorname{rank}\left\{\mathbf{B}_{r}\right\}-P \\
& \leq \operatorname{rank}\left\{\mathbf{T}_{a} \mathbf{B}_{r}\right\} \leq \min \left(\operatorname{rank}\left\{\mathbf{T}_{a}\right\}, \operatorname{rank}\left\{\mathbf{B}_{r}\right\}\right)
\end{aligned}
$$

With $P=L$, it is easy to see that in the most general case (i.e., for distinct generators $\left.\left\{b_{j}\right\}_{j=1}^{P}\right)$, the Vandermonde matrix $\mathbf{B}_{r}$ is of rank $L-1$. Equation (A.1) can then be rewritten as

$$
(L-1) \leq \operatorname{rank}\left\{\mathbf{T}_{a} \mathbf{B}\right\} \leq(L-1) .
$$

In other words, the matrix $\mathbf{H}_{b}(\boldsymbol{\Omega}) \in \mathbb{C}^{K(L-1) \times L}$ does not have full $\operatorname{rank}\left\{\mathbf{H}_{b}(\boldsymbol{\Omega})\right\}=\operatorname{rank}\left\{\mathbf{T}_{a} \mathbf{B}\right\}=L-1<L$ which contradicts Assumption 3. Similarly we can prove that $M_{b}<K$ is necessary for $\mathbf{H}_{a}(\boldsymbol{\Omega})$ to be nonsingular. Further it is simple to show that the validity of Assumption 3 implies that also Assumption 2 is satisfied.

\section{B. PROOF OF PROPOSITION 1}

In order to prove that $\mathbf{G}_{a}$ has full column rank, it is sufficient to consider the limiting case $P=L(K-1)$ where $\mathbf{G}_{a}$ becomes a square matrix. The proof is based on the application of appropriate elementary matrix operations applied on the rows of $\mathbf{G}_{a}$. More precisely, we exploit that adding a multiple of the row of a matrix to any other row does not change the determinant of the matrix. Similar to the procedure used in Gaussian elimination, we wish to bring the first $L$ columns of $\mathbf{G}_{a}$ into "triangular" form. Towards this aim, we subtract $a$ times the $(k-1)$ th row of $\mathbf{G}_{a}$ from the $k$ th row of $\mathbf{G}_{a}$, for $k=2, \ldots, K, K+2, \ldots, 2 K, 2 K+2, \ldots, 3 K, \ldots,(L-1) K,(L-$ $1) K+2, \ldots, L K$, that is, for all $k \in\{1, \ldots, K L\}$ such that $(k)_{K} \neq 1$, where $(k)_{K}$ denotes $k$ modulo $K$. The $k$ th row of the resulting matrix denoted by $\overline{\mathbf{G}}_{a}$ is given by

$$
[\underbrace{0, \ldots, 0}_{L} \mid \underbrace{b_{1}^{\lfloor k / K\rfloor} a_{1}^{\left((k)_{K}-2\right)}\left(a_{1}-a\right), \ldots, b_{P}^{\lfloor k / K\rfloor} a_{P}^{\left((k)_{K}-2\right)}\left(a_{P}-a\right)}_{P}]
$$

for $(k)_{K} \neq 1$. For $(k)_{K}=1$, the rows of $\overline{\mathbf{G}}_{a}$ remain unchanged and identical to the corresponding rows of $\mathbf{G}_{a}$. Note that $\operatorname{det}\left\{\overline{\mathbf{G}}_{a}\right\}=\operatorname{det}\left\{\mathbf{G}_{a}\right\}$. It can readily be verified that each of the $L$ first columns of $\overline{\mathbf{G}}_{a}$ contain only a single nonzero element. These columns form a matrix $\mathbf{T}_{0}=\mathbf{T}_{a \mid a=0}=$ $\left[\mathbf{e}_{1}, \mathbf{e}_{K+1}, \mathbf{e}_{2 K+1}, \ldots, \mathbf{e}_{(L-1) K+1}\right]$ where $\mathbf{e}_{k}$ denotes the $k$ th column of a $K L \times K L$ identity matrix $\mathbf{I}_{K L}$. Making use of a wellknown expansion rule for determinants, it is immediate to show that

$$
\begin{aligned}
\operatorname{det}\left\{\mathbf{G}_{a}\right\} & =\operatorname{det}\left\{\overline{\mathbf{G}}_{a}\right\}=\operatorname{det}\left\{\left[\mathbf{T}_{0} \mid \mathbf{H}_{a}(\boldsymbol{\Omega}) \boldsymbol{\Delta}_{a}\right]\right\} \\
& = \pm \operatorname{det}\left\{\mathbf{H}_{a}(\boldsymbol{\Omega})\right\} \operatorname{det}\left\{\boldsymbol{\Delta}_{a}\right\} \\
& = \pm \operatorname{det}\left\{\mathbf{H}_{a}(\boldsymbol{\Omega})\right\} \prod_{p=1}^{P}\left(a_{p}-a\right),
\end{aligned}
$$

where $\Delta_{a}=\operatorname{diag}\left\{\left[\left(a_{1}-a\right), \ldots,\left(a_{P}-a\right)\right]\right\}$ and " \pm " indicates that equality holds up to “+” or "-" sign. Provided that $\mathbf{H}_{a}(\boldsymbol{\Omega})$ has full rank, we observe from (B.2) that for $a \neq a_{p},(p=1, \ldots, P, P \leq L(K-1))$ the determinant $\operatorname{det}\left\{\mathbf{G}_{a}\right\} \neq 0$ and $\operatorname{det}\left\{\mathbf{G}_{a}\right\}=0$, otherwise. For $\mathbf{G}_{b}$ the proof follows in a similar manner from (B.2).

\section{PROOF OF COROLLARY 1}

Without loss of generality, we assume that $a=a_{1}, \ldots, a_{M_{a}}$ is a true generator of multiplicity $M_{a} \leq K$ that is associated with the first $M_{a}$ harmonics, that is, the first $M_{a}$ columns of $\mathbf{H}(\boldsymbol{\Omega})$ (See Remark 2). From (B.2) we conclude that ma$\operatorname{trix} \mathbf{H}(\boldsymbol{\Omega})^{H}\left(\mathbf{I}_{K L}-\mathbf{T}_{a}\left(\mathbf{T}_{a}^{H} \mathbf{T}_{a}\right)^{-1} \mathbf{T}_{a}^{H}\right) \mathbf{H}(\boldsymbol{\Omega})$ in (16) has exactly $M_{a}$ zero eigenvalues $\mu_{1,0}=\cdots, \mu_{M_{a}, 0}=0$. Furthermore, the eigenvectors corresponding to the zero eigenvalues are equivalent to the first $M_{a}$ columns of a $P \times P$ identity matrix. The last property follows from the fact that (B.2) and consequently (16) hold true for any choice of harmonics with $P \leq L(K-1)$ including the single harmonic case, where $P=1$ and $\mathbf{H}(\boldsymbol{\Omega})=\mathbf{h}\left(a_{1}, b_{1}\right)=\mathbf{b}_{1} \circ \mathbf{a}_{1}$. This observation implies that in the multiharmonic case, and with $\mathbf{h}\left(a_{p}, b_{p}\right)$ denoting the $p$ th column of the signal matrix $\mathbf{H}(\boldsymbol{\Omega})$ identity,

$$
\begin{aligned}
F_{R, a}(a) & =\mathbf{h}^{H}\left(a_{p}, b_{p}\right)\left(\mathbf{I}_{K L}-\mathbf{T}_{a_{p}}\left(\mathbf{T}_{a_{p}}^{H} \mathbf{T}_{a_{p}}\right)^{-1} \mathbf{T}_{a_{p}}^{H}\right) \mathbf{h}\left(a_{p}, b_{p}\right) \\
& =\mathbf{e}_{p}^{H} \mathbf{H}(\boldsymbol{\Omega})^{H}\left(\mathbf{I}_{K L}-\mathbf{T}_{a_{p}}\left(\mathbf{T}_{a_{p}}^{H} \mathbf{T}_{a_{p}}\right)^{-1} \mathbf{T}_{a_{p}}^{H}\right) \mathbf{H}(\boldsymbol{\Omega}) \mathbf{e}_{p} \\
& =0
\end{aligned}
$$

holds true for $p=1, \ldots, M_{a}$. That is for $a=a_{1}, \ldots, a_{M_{a}}$ and $M_{a} \leq K$, the unit vectors $\left\{\mathbf{e}_{p}\right\}_{p=1}^{M_{a}}$ form an orthogonal basis for the nullspace of $\mathbf{H}(\boldsymbol{\Omega})^{H}\left(\mathbf{I}_{K L}-\mathbf{T}_{a}\left(\mathbf{T}_{a}^{H} \mathbf{T}_{a}\right)^{-1} \mathbf{T}_{a}^{H}\right) \mathbf{H}(\boldsymbol{\Omega})$. With $\mathbf{H}(\boldsymbol{\Omega})=\mathbf{E}_{S} \mathbf{K}$, it is immediate that the vectors $\left\{\tilde{\boldsymbol{\gamma}}_{p, 0}=\right.$ $\left.\mathbf{K e}_{p}=\mathbf{k}_{p}\right\}_{p=1}^{M_{a}}$ span the nullspace of $\mathbf{M}(a)$ (19) denoted by $\mathcal{N}\{\mathbf{M}(a)\}$.

Similarly, assuming $b=b_{1}, \ldots, b_{M_{b}}$ to denote a true generator of multiplicity $M_{b} \leq L$, we obtain that the vectors $\left\{\tilde{\boldsymbol{\gamma}}_{p, 0}=\mathbf{K e}_{p}=\mathbf{k}_{p}\right\}_{p=1}^{M_{b}}$ span the nullspace $\mathcal{N}\{\mathbf{M}(b)\}$ (21). Since by Assumption 2 all 2D harmonics can uniquely be recovered from (8), at least one of the generators $a_{p}$ and $b_{p}$ of a specific generator pair $\left(a_{p}, b_{p}\right)$ is of multiplicity one. Hence, we conclude that for a true generator pair $\left(a_{p}, b_{p}\right)$, the associated nullspaces $\mathcal{N}\left\{\mathbf{M}\left(a_{p}\right)\right\}$ and $\mathcal{N}\left\{\mathbf{M}\left(b_{p}\right)\right\}$ share exactly one common nullspace vector given, for example, by $\mathbf{k}_{p}$. Moreover, the two nullspaces do not intersect if $a_{p}$ and $b_{p}$ solve the individual RARE polynomial equations (20) and (22) but $\left(a_{p}, b_{p}\right)$ does not correspond to a true generator pair. That is, for a true generator pair $\left(a_{p}, b_{p}\right)$, the vector $\mathbf{k}_{p}$ marks the intersection of the nullspaces $\mathcal{N}\left\{\mathbf{M}\left(a_{p}\right)\right\}$ and $\mathcal{N}\left\{\mathbf{M}\left(b_{p}\right)\right\}$ while the nullspaces do not intersect otherwise. It immediately follows that Corollary 1 holds true for arbitrary $0<\alpha<1$. 


\section{ACKNOWLEDGMENTS}

The authors would like to thank Steffen Paul, Infineon Technologies, for valuable comments on this work and Ernst Bonek for continuous encouragement and support. The Weikendorf measurements were carried out under the supervision of Helmut Hofstetter, FTW Part of this work was carried out with funding from Kplus in FTW Project C3 "Smart Antennas for UMTS Frequency Division Duplex" together with Infineon Technologies and Austrian Research Centers (ARCS), Seibersdorf.

\section{REFERENCES}

[1] M. Steinbauer, D. Hampicke, G. Sommerkorn, et al., "Array measurement of the double-directional mobile radio channel," in Proc. IEEE 51nd Annual Vehicular Technology Conference, vol. 3, pp. 1656-1662, Tokyo, Japan, May 2000.

[2] R. S. Thomä, D. Hampicke, A. Richter, G. Sommerkorn, A. Schneider, and U. Trautwein, "Identification of timevariant directional mobile radio channels," in Proc. IEEE 16th Instrumentation and Measurement Technology Conference, vol. 1, pp. 176-181, Venice, Italy, May 1999.

[3] M. Haardt and J. A. Nossek, "Simultaneous Schur decomposition of several nonsymmetric matrices to achieve automatic pairing in multidimensional harmonic retrieval problems," IEEE Trans. Signal Processing, vol. 46, no. 1, pp. 161169, 1998.

[4] T. Jiang, N. D. Sidiropoulos, and J. M. F. ten Berge, "Almostsure identifiability of multidimensional harmonic retrieval," IEEE Trans. Signal Processing, vol. 49, no. 9, pp. 1849-1859, 2001.

[5] M. Pesavento, A. B. Gershman, and K. M. Wong, "Direction finding in partly calibrated sensor arrays composed of multiple subarrays," IEEE Trans. Signal Processing, vol. 50, no. 9, pp. 2103-2115, 2002.

[6] H. L. Van Trees, Detection, Estimation, and Modulation Theory, Part IV: Optimum Array Processing, John Wiley \& Sons, New York, NY, USA, 2002.

[7] R. Roy and T. Kailath, "ESPRIT-estimation of signal parameters via rotational invariance techniques," IEEE Trans. Acoustics, Speech, and Signal Processing, vol. 37, no. 7, pp. 984-995, 1989.

[8] M. Pesavento, A. B. Gershman, K. M. Wong, and J. F. Böhme, "Direction finding in partly calibrated arrays composed of nonidentical subarrays: a computationally efficient algorithm for the rank reduction (RARE) estimator," in Proc. IEEE 11th Statistical Signal Processing Workshop, pp. 536-539, Orchid Country Club, Singapore, August 2001.

[9] C. P. Mathews and M. D. Zoltowski, "Eigenstructure techniques for 2-D angle estimation with uniform circular arrays," IEEE Trans. Signal Processing, vol. 42, no. 9, pp. 2395-2407, 1994.

[10] H. Hofstetter, I. Viering, and W. Utschick, "Evaluation of suburban measurements by eigenvalue statistics," in Proc. 1st COST 273 Workshop and 4th Management Committee Meeting, Espoo, Finland, May 2002.

[11] H. Hofstetter, C. F. Mecklenbräuker, R. Müller, et al., "Description of wireless MIMO measurements at $2 \mathrm{GHz}$ in selected environments," in COST-273 5th Meeting, TD(02)135, Lisbon, Portugal, September 2002.

[12] G. Sommerkorn, D. Hampicke, R. Klukas, A. Richter, A. Schneider, and R. S. Thomä, "Uniform rectangular antenna array design and calibration issues for 2-D ESPRIT applica- tion," in Proc. 4th European Personal Mobile Communications Conference, Vienna, Austria, February 2001.

Marius Pesavento was born in Werl, Germany, in 1973. He received the Dipl.-Ing. degree in electrical engineering from RuhrUniversität Bochum, Germany, in 1999. From 1999 to 2000, he was with the Department of Electrical and Computer Engineering, McMaster University, Hamilton, Ontario, Canada, where he received his M.Eng. degree (with highest honors). He is currently with the Department of Electrical En-

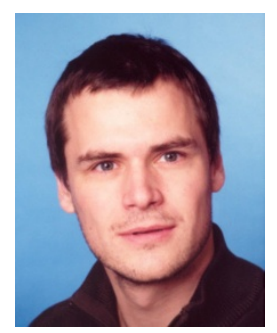
gineering, Ruhr-Universität Bochum, where he is pursuing his Ph.D. degree. His research intersts include statistical signal and array processing, adaptive beamforming, and parameter estimation. Mr. Pesavento was a recipient of the 2001 Outstanding Thesis Research Award from McMaster University and the 2003 ITG best paper award from the Association of Electrical Engineering, Electronics, and Information Technologies (VDE).

Christoph F. Mecklenbräuker was born in Darmstadt, Germany, in 1967. He received the Dipl.-Ing. degree in electrical engineering from Vienna University of Technology in 1992 and the Dr.-Ing. degree from RuhrUniversität Bochum in 1998, respectively. His doctoral thesis on matched field processing was awarded the Gert Massenberg Prize. He worked for the Mobile Networks Radio Department of Siemens AG where he

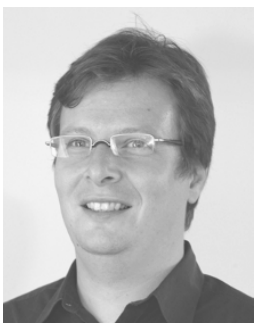
participated in the European framework of ACTS 90 "FRAMES." He was a delegate to the Third Generation Partnership Project (3GPP) and engaged in the standardization of the radio access network for UMTS. Since 2000, he has been holding a senior research position at the Telecommunications Research Center Vienna (FTW) in the field of mobile communications. Currently, he gives a course at the Vienna Technical University on 3G mobile networks. He has authored around 60 papers in international journals and conferences, for which he has also served as a reviewer and holds 8 patents in the field of mobile cellular networks. His current research interests include antenna-array- and MIMO-signal processing for mobile communications.

Johann F. Böhme was born in Senftenberg, Germany on January 26, 1940. He received the Diploma degree in mathematics in 1966, the Dr.-Ing. in 1970, and the Habilitation in 1977, all in computer science, from the Technical University of Hanover, Germany, the University of Erlangen, Germany, and the University of Bonn, Germany, respectively. From 1967 to 1974, he was with the sonar-research laboratory of

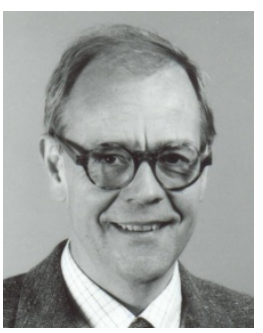
Krupp Atlas Elektronik in Bremen, Germany. He then joined the University of Bonn until 1978 and the FGAN in WachtbergWerthhoven. Since 1980, he has been Professor of signal theory in the Department of Electrical Engineering and Information Sciences at Ruhr-Universität Bochum, Germany. His research interests are in the domain of statistical signal processing and its applications. He is a Fellow of the Institution of Electrical and Electronic Engineers and an Elected Member of the North Rhine-Westphalian Academy of Sciences. 